\title{
The Berry phase in graphene and graphite multilayers
}

\author{
G.P. Mikitik and Yu.V. Sharlai \\ B. Verkin Institute for Low Temperature Physics and Engineering of the National Academy of Sciences of Ukraine \\ 47 Lenin Ave., Kharkov 61103, Ukraine \\ E-mail: mikitik@ilt.kharkov.ua \\ E-mail: sharlai@ilt.kharkov.ua
}

Received March 28, 2008

\begin{abstract}
We discuss the electron energy spectra and the Berry phases for graphene, a graphite bilayer, and bulk graphite allowing for a small spin-orbit interaction. If an electron orbit in the Brillouin zone surrounds several Dirac points (band-contact lines in graphite), one can find relative signs of the Berry phases generated by these points (lines) by taking into account this interaction.
\end{abstract}

PACS: 73.22.Dj Single particle states;

03.65.Sq Semiclassical theories and applications ;

03.65.Vf Phases: geometric; dynamic or topological.

Keywords: Berry phase, graphite bilayer.

\section{Introduction}

In the last few years graphene, i.e. a graphite monolayer, has attracted considerable attention thanks to its unusual physical properties, see, e.g., the recent review [1] and references therein. In particular, a phase shift of the Shubnikov-de Haas oscillations and a half-integer quantum Hall effect were observed in this two-dimensional material $[2,3]$. These properties of graphene are due to the specific feature of its electron energy spectrum: At the point $\mathrm{K}$ of its Brillouin zone the electron and hole bands $\varepsilon_{e, h}(\mathbf{k})$ touch one another, while in the vicinity of this point they split linearly in the wave vector $\mathbf{k}$, see Fig. 1. In other words, this spectrum looks like the spectrum of relativistic Dirac particles, and so the band-contact point in graphene received the name Dirac point. This type of the spectrum generates a nonzero value

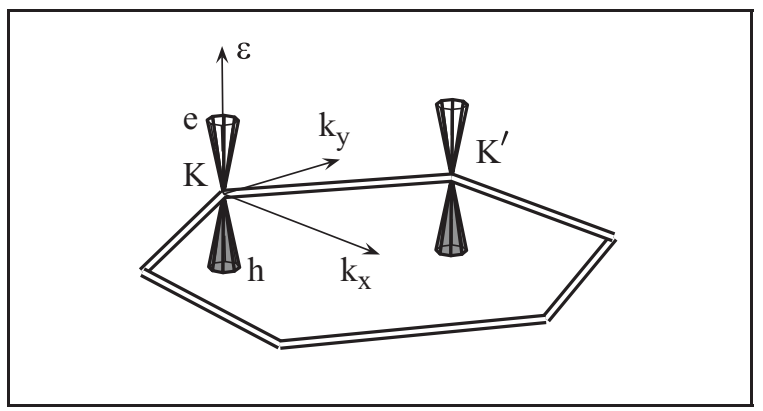

Fig. 1. The dispersion law $\varepsilon\left(k_{x}, k_{y}\right)$ of the electrons (e) and holes (h) in graphene near the point $K$ of its hexagonal Brillouin zone. of the so-called Berry phase [4], the Berry phase modifies the semiclassical quantization condition for the electron energy in a magnetic field [5], and this modification leads to a shift of the Landau levels in graphene as compared to the levels for the usual parabolic spectrum. It is this shift of the Landau levels that explains the above-mentioned properties of graphene. One may expect that these considerations based on the semiclassical approach fail for the lowest Landau levels, i.e., when the quantum number $n \sim 1$. But in the case of linear dependence $\varepsilon(\mathbf{k})$ the electron spectrum in a magnetic field can be found exactly, see, e.g., Refs. 6,7, and it is turned out that the exact and semiclassical spectra coincide for all $n$, including $n=0$ [6]. Thus, in the case of graphene one can apply considerations based on the Berry phase without any restrictions.

In this paper we first review results concerning the Berry phase in metals, and then discuss the Berry phase in graphene, in a graphite bilayer, and in a bulk graphite that can be considered as a sample with a sufficiently large number of the layers. Moreover, in this paper we shall analyze the Berry phase taking into account the spin-orbit interaction since this interaction is important for understanding of the Berry phase in graphite multilayers.

\section{The Berry phase in metals}

According to Berry [4], if a Hamiltonian of a quantum system depends on parameters, and if the parameters undergo adiabatic changes so that they eventually return to 
their original values, the wave function of the system can acquire the so-called geometrical phase in addition to the familiar dynamical one. This additional phase (the Berry phase) differs from zero when the trajectory $\Gamma$ of the system in the parameter space is located near a point at which the states of the system are degenerate. In analyzing this situation, Berry assumed that the Hamiltonian of the system is a Hermitian matrix which is linear in deviations of the parameters from the point, and he presented his final result in the pictorial form. He found that such the point can be considered as a «monopole» in the parameter space when the geometrical phase is calculated. In other words, the point «generates» a field which coincides in the form with that of the monopole, and the flux of this Berry field through the contour $\Gamma$ gives the geometrical phase of the system.

Berry's result is applicable to the semiclassical electron trajectories in crystals, with the Brillouin zone playing the role of the parameter space [8]. However, in three-dimensional crystals with the inversion symmetry and a weak spin-orbit interaction, the Berry phase of the electrons has the specific features [5] which are due to the fact that the electron states are invariant under the simultaneous inversion of time and spatial coordinates. This invariance permits one to transform the Hermitian Hamiltonian of the electron into the real form for any point of the Brillouin zone. As a consequence, the character of the energy-band degeneracy in the three-dimensional crystals differs from that considered by Berry. Now the electron energy bands $\varepsilon_{l}(\mathbf{k})$ contact along lines in the Brillouin zone rather than at points, and the lines need not be symmetry axes [9]. In other words, Berry's monopoles in the $\mathbf{k}$ space disappear. However, as it was shown in our paper [5], the above-mentioned band-contact lines play the role of infinitely thin «solenoids» which generate the Berry field with the flux $\pm \pi$, and the situation similar to the Aharonov-Bohm effect [10] occurs: Although this field is zero outside the solenoids, but if the electron orbit surrounds a contact line of its band with some other band, the flux threads the orbit, and the electron acquires the Berry phase $\Phi_{B}= \pm \pi$ when it moves around this line. It is clear that in this case the Berry phase does not depend on the shape and the size of the electron orbit but is specified only by its topological characteristics (there is a linking of the orbit with the band-contact line or not). In the case of a two-dimensional metal the band-contact lines transform into the Dirac points, and if the electron orbit $\Gamma$ surrounds Dirac points, each of these points contributes $\pm \pi$ to $\Phi_{B}$. When there are no Dirac points inside the orbit, the Berry phase is equal to zero.

The Berry phase is defined up to $2 \pi$, and in this context the phases $\Phi_{B}=\pi$ and $\Phi_{B}=-\pi$ are equivalent. But when the electron orbit surrounds several of the band-contact lines (the Dirac points), it may be useful to know relative signs of the Berry phases generated by each of the line (the Dirac point). This can be done using the approach of Ref. 11 in which the effect of a small spin-orbit interaction on the Berry phase was investigated. It is important that if this interaction is weak, it does not change the Berry phase, but it fixes the sign of the phase for a given direction of the electron spin [11,12]. Here we present the formulas that enable one to calculate the Berry phase $\Phi_{B}$ in this case.

Let an electron be in the band $\varepsilon_{0}(\mathbf{k})$. Consider an orbit $\Gamma$ of this electron in the Brillouin zone. The Berry phase for the orbit $\Gamma$ and at a fixed direction of the electron spin is given by $[5,8]$

$$
\Phi_{B}=\oint_{\Gamma} \Omega d \mathbf{k},
$$

where $\Omega$ is the intraband matrix element of the periodic (in $\mathbf{k}$ ) part of the coordinate operator in the crystal momentum representation [13],

$$
\boldsymbol{\Omega}(\mathbf{k})=i \int d \mathbf{r} u_{\mathbf{k} 0}^{*}(\mathbf{r}) \nabla_{k} u_{\mathbf{k} 0}(\mathbf{r}),
$$

and $u_{\mathbf{k} 0}(\mathbf{r})$ is the periodic factor in the electron Bloch wave function of the band $0, \psi_{\mathbf{k} 0}(\mathbf{r})=\exp (i \mathbf{k} r) u_{\mathbf{k} 0}(\mathbf{r})$. Note that the Berry phase changes its sign for the opposite direction of the electron spin since it follows from symmetry considerations [11] that $\boldsymbol{\Omega}_{\mathrm{up}}=-\boldsymbol{\Omega}_{\mathrm{down}}$ for the spin directed «up» and «down».

The Berry phase manifests itself in the Onzager-Lifshitz-Kosevich quantization condition [14,15] for the Landau levels $\varepsilon_{n}$ of the electron in the magnetic field $\mathbf{H}$,

$$
S\left(\varepsilon_{n}, k_{H}\right)=\frac{2 \pi e H}{\hbar c}(n+\gamma),
$$

where $S$ is the cross-section area of the closed orbit in the $\mathbf{k}$ space; $k_{H}$ is the component of $\mathbf{k}$ along the magnetic field $\mathbf{H} ; n$ is a large integer $(n>0) ; e$ is the absolute value of the electron charge, and the constant $\gamma$ is given by the formula [5]:

$$
\gamma=\frac{1}{2}-\frac{\Phi_{B}}{2 \pi} .
$$

The direction of the integration in Eq. (1) is now determined by the vector $\operatorname{sign}\left(m_{*}\right)\left[\mathbf{H} \times \partial \varepsilon_{0} / \partial \mathbf{k}\right]$ where

$$
m_{*}=\frac{\hbar^{2}}{2 \pi} \frac{\partial S\left(\varepsilon, k_{H}\right)}{\partial \varepsilon}
$$

is the cyclotron mass which is positive for electrons and negative for holes, and hence $\operatorname{sign}\left(m_{*}\right)= \pm 1$. The direction of the integration becomes essential when one wants to determine not only the value of the Berry phase but also its sign.

The meaning of formula (4) is simple: When the electron makes a complete circuit in its orbit, the change of 
the phase of its wave function consists of the usual semiclassical part $\hbar c S / e H$, the shift $-\pi$ associated with the so-called turning points of the orbit where the semiclassical approximation fails, and the Berry phase. Equating this change to $2 \pi n$, one arrives at Eqs. (3), (4). Thus, when the electron orbit links to the band-contact line, one obtains $\gamma=0$ or $\gamma=1$ instead of the usual value $\gamma=1 / 2$. Note that the quantity $-\Phi_{B} / 2 \pi$ in Eq. (4), in fact, describes the electron $g$ factor that differs from zero even at infinitesimal spin-orbit interaction if the orbit surrounds the bandcontact line (the Dirac point) [16].

The quantity $\Omega$ can be calculated for any electron-band structure if an appropriate $\mathbf{k} \cdot \mathbf{p}$ Hamiltonian is known [11]:

$$
\boldsymbol{\Omega}=i\left(S^{+} \frac{\partial S}{\partial \mathbf{k}}\right)_{00},
$$

where $S(\mathbf{k})$ is the matrix reducing the $\mathbf{k} \cdot \mathbf{p}$ Hamiltonian to the diagonal form, $S^{+}$is the Hermitian conjugate matrix, and the subscript means that one has to consider the diagonal matrix element corresponding to the band 0 discussed here. This formula follows from Eq. (2) if one takes into account the definition of the matrix $S$,

$$
S_{i j}(\mathbf{k})=\int u_{0 i}^{*}(\mathbf{r}) u_{\mathbf{k} j}(\mathbf{r}) d \mathbf{r} .
$$

As it is well known [17], a frequency of de Haas-van Alphen oscillations, or of other oscillation effects in the magnetic field, gives an extremal cross-section area $S_{\text {ex }}$ of the Fermi surface of a metal. On the other hand, a phase of these oscillations is expressed via the constant $\gamma$ of the semiclassical quantization rule. For example, the first harmonic of the de Haas-van Alphen oscillations of the magnetic susceptibility has the form [15],

$$
\chi \cos \left(\frac{\hbar c S_{\mathrm{ex}}}{e H}+\varphi\right)
$$

where $S_{\text {ex }}$ is some extremal (in $k_{H}$ ) cross section of the Fermi surface of a metal, a positive $\chi$ is the amplitude of this first harmonic, and $\varphi$ is its phase which is given by

$$
\varphi=-2 \pi \gamma+\delta
$$

with $\delta= \pm \pi / 4$ for a minimum and maximum cross-section $S_{\text {ex }}$, respectively, and $\delta=0$ in the case of a two-dimensional Fermi surface. The phase $\varphi$ (together with the frequency) can be found by the Fourier analysis of the magnetic-susceptibility oscillations [18] or by plotting the fan diagram [17]. Thus, a measurement of the phase of the de Haas-van Alphen oscillations gives $\gamma$ and hence enables one to detect a band-contact line if it penetrates the extremal cross section of the Fermi surface even though this line lies far away from the Fermi level.

In two-dimensional metals, when the quantum Hall effect is observable, the Berry phase also manifests itself in the Hall conductivity. In the conventional integer quan- tum Hall effect, when the filling factor $v=h c N /(e g H)$ of the Landau levels reaches an integer value $n$, the Hall conductivity $\sigma_{x y}(v)$ shows the plateau that is equal to $\sigma_{x y}=$ $=\left(g e^{2} / h\right) n$. Here $N$ is the two-dimensional density of electrons in a metal, and $g$ is the integer accounting for a degeneracy of the electron spectrum (in graphene $g=4$ due to two points $K$ in the Brillouin zone and the two-fold spin degeneracy). The nonzero Berry phase changes the constant $\gamma$ and hence shifts the Landau levels in the energy scale. This shift leads to a half-integer quantum Hall effect: The plateaus of $\sigma_{x y}(n)$ now occurs at $\sigma_{x y}=$ $=\left(g e^{2} / h\right)(n+1 / 2)$.

\section{The Berry phase in grapheme}

Let us now consider the Berry phase in graphene taking into account the spin-orbit interaction. The effect of the spin-orbit interaction on the spectrum of graphene was considered in Refs. 19,20, and the following $\mathbf{k} \cdot \mathrm{p}$ Hamiltonian for the electrons and holes with spin up was obtained in the vicinity of the point $\mathrm{K}$ of the Brillouin zone:

$$
\hat{H}=\hbar v\left(k_{x} \sigma_{x}+k_{y} \sigma_{y}\right)+\Delta_{s o} \sigma_{z},
$$

where $\sigma_{i}$ are the Pauli matrices describing the two bands, $v$ is a matrix element of the velocity operator, $v \approx 10^{6} \mathrm{~m} / \mathrm{s}$, and $2 \Delta_{s o} \sim 0.2 \mathrm{meV}$ is the gap caused by the spin-orbit interaction. This Hamiltonian leads to the following dispersion laws $\varepsilon_{e, h}(\mathbf{k})$ for the electrons (e) and holes (h) in graphene:

$$
\varepsilon_{e, h}(\mathbf{k})= \pm \sqrt{\Delta_{s o}^{2}+(\hbar v k)^{2}},
$$

where the signs plus and minus correspond to the electrons and holes, respectively.

Hamiltonian (8) can be transformed to the diagonal form (9) by the matrix

$$
\hat{S}=\frac{1}{\sqrt{2 \varepsilon_{e}\left(\varepsilon_{e}+\Delta_{s o}\right)}}\left(\begin{array}{cc}
\Delta_{s o}+\varepsilon_{e} & \hbar v k_{-} \\
\hbar v k_{+} & -\left(\Delta_{s o}+\varepsilon_{e}\right)
\end{array}\right)
$$

where $k_{ \pm}=k_{x} \pm i k_{y}$. Then, formula (5) yields

$$
\Omega_{x}=\frac{\hbar^{2} v^{2} k_{y}}{2 \varepsilon_{e}\left(\varepsilon_{e}+\Delta_{s o}\right)}, \Omega_{y}=-\frac{\hbar^{2} v^{2} k_{x}}{2 \varepsilon_{e}\left(\varepsilon_{e}+\Delta_{s o}\right)},
$$

Inserting this $\Omega$ into Eq. (1), we obtain the Berry phase

$$
\Phi_{B}=\oint_{\Gamma} \Omega d \mathbf{k}=-\pi\left(1-\frac{\Delta_{s o}}{\varepsilon_{e}}\right)
$$

for an electron orbit $\Gamma$ that is defined by the condition $\varepsilon_{e}(\mathbf{k})=$ const $>0$. Note that this expression agrees with the intraband contribution to the electron $g$ factor calculated for a more general two-band model of the spectrum in Ref. 11. Finally, in the limit of the infinitesimal spin-orbit interaction we arrive at

$$
\Phi_{B}=\lim _{\Delta_{s o} \rightarrow 0} \Phi_{B}\left(\Delta_{s o}, \varepsilon_{e}\right)=-\pi .
$$


In similar manner one obtains $\Phi_{B}=\pi$ for the holes with spin up, while for the quasiparticles with spin down the Berry phase changes its sign.

Formula (4) gives $\gamma=1$ for the electrons and $\gamma=0$ for the holes with the spin up. Inserting the expression for the cross-section area $S=\pi\left(E^{2}-\Delta_{s o}^{2}\right) /(\hbar v)^{2}$ into Eq. (3), and using the obtained values of $\gamma$, we arrive at the spectrum in the magnetic field $H$ [21]:

$$
\varepsilon_{n}= \pm \sqrt{\Delta_{s o}^{2}+\frac{2 e \hbar v^{2} H n}{c}},
$$

where \pm correspond to the electrons and holes, respectively, and $n$ goes through the values $1, \ldots \infty$ for the electrons and $0, \ldots \infty$ for the holes. For the spin down the spectrum is still given by Eq. (14), but the intervals of admissible $n$ for the electrons and holes are exchanged. Thus, the lowest electron Landau level with spin down lies just at the edge $\Delta_{s o}$ of the upper band, while the edge $-\Delta_{s o}$ of the hole band coincides with the Landau level with spin up. All other Landau level are two-fold degenerate in spin. We emphasize that the spectrum thus obtained is exact, i.e., it is valid at all integer $n$, including $n=0[6]$.

If the spin-orbit gap $\Delta_{s o}$ tends to zero, one finds that all the Landau levels are two-fold degenerate, and they can be described by Eq. (3) with $\gamma=0$. The Landau level $n=0$ now lies exactly at $\varepsilon=0$ and it is shifted in energy as compared to the the lowest Landau level for a parabolic spectrum. As was mentioned above, this shift leads to the half-integer quantum Hall effect that was indeed observed in graphene in Refs. 2,3. In these papers the constant $\gamma$ was also measured using Shubnikov-de Haas oscillations, and the value $\gamma=0$ was obtained.

\section{The Berry phase in a graphite bilayer}

In the recent letter [22] McCann and Fal'ko considered the electron-energy spectrum of a graphite bilayer in the vicinity of the point $\mathrm{K}$ of its Brillouin zone and stated that in this bilayer the low-energy electronic excitations correspond to chiral quasiparticles with a parabolic dispersion exhibiting Berry phase $2 \pi$.

First of all we point out that the electron spectrum of the bilayer [22] can be easily obtained from the well-known Slonzewski-Weiss-McClure (SWM) spectrum [23] of bulk graphite if one puts $\cos \xi=0.5$ and $\gamma_{2}=\gamma_{5}=0$ (the parameters $\gamma_{2}, \gamma_{5}$ describe the interaction of the atoms in the next-nearest-neighbor layers of graphite that are absent in the bilayer; $\xi$ is the dimensionless wave vector perpendicular to the graphite layers). The SWM model describes the wave-vector dependence of four electron energy bands of graphite $\varepsilon(\mathbf{k})$ in the vicinity of the vertical edge $H K H$ of its Brillouin zone. These bands can be found from the forth-order secular equation:

$$
\operatorname{det}|\hat{H}-\varepsilon|=0,
$$

where the Hamiltonian matrix $\hat{H}$ has the form

$$
\hat{H}=\left(\begin{array}{cccc}
E_{1} & 0 & H_{13} & H_{13}^{*} \\
0 & E_{2} & H_{23} & -H_{23}^{*} \\
H_{13}^{*} & H_{23}^{*} & E_{3} & H_{33} \\
H_{13} & -H_{23} & H_{33}^{*} & E_{3}
\end{array}\right) .
$$

Here the following notations have been used:

$$
\begin{aligned}
E_{1} & =\Delta+\gamma_{1} \Gamma+\frac{1}{2} \gamma_{5} \Gamma^{2}, \\
E_{2} & =\Delta-\gamma_{1} \Gamma+\frac{1}{2} \gamma_{5} \Gamma^{2}, \\
E_{3} & =\frac{1}{2} \gamma_{2} \Gamma^{2}, \\
H_{13} & =\frac{1}{\sqrt{2}}\left(-\gamma_{0}+\gamma_{4} \Gamma\right) \mathrm{e}^{i \alpha} \zeta, \\
H_{23} & =\frac{1}{\sqrt{2}}\left(\gamma_{0}+\gamma_{4} \Gamma\right) \mathrm{e}^{i \alpha} \zeta, \\
H_{33} & =\gamma_{3} \Gamma \mathrm{e}^{i \alpha} \zeta,
\end{aligned}
$$

where $\alpha$ is the angle between the direction of the vector $\mathbf{k}$ and the $K \Gamma$ direction in the Brillouin zone; $\Gamma=2 \cos \xi ; \xi$ and $\zeta$ are dimensionless wave vectors in the direction of the $z$-axis (i.e. HKH axis) and in the basal plane, respectively: $\xi=(\pi / 2)\left(k_{z} /|K H|\right), \zeta=(2 \pi / \sqrt{3}) \times$ $\times\left(k_{\perp} /|\Gamma K|\right) ; k_{\perp}=\sqrt{k_{x}^{2}+k_{y}^{2}} ; \mathbf{k}$ is measured from the point $\mathrm{K} ;|\Gamma K|$ and $|K H|$ are the distances between the point $\mathrm{K}$ and points $\Gamma$ and $\mathrm{H}$ of the Brillouin zone. The parameter $\gamma_{0}$ which describes the interaction between neighbor atoms in a graphite layer is sufficiently large as compared to the other parameters $\gamma_{i}, \Delta$ which describe relatively weak interactions between atoms in different graphite layers. If one puts $\gamma_{i}=\Delta=0(i=1-5)$ and $\gamma_{0}==\sqrt{3} \hbar v|\Gamma K| / 2 \pi$, we arrive at the double degenerate spectrum of graphene (9) with $\Delta_{s o}=0$. On the other hand, the spectrum of the bilayer is obtained if one puts $\Gamma=1$ and $\gamma_{2}=\gamma_{5}=0$, see Table 1. Note that in this way one can allow for the small parameter $\gamma_{4} \approx 0.044 \mathrm{eV}$ that was neglected in Ref. 22 .

Table 1. Values of the parameters of SWM model $(\mathrm{eV})$ that lead to the spectrum of Ref. 22.

\begin{tabular}{c|c}
\hline \hline Parameter & Value \\
\hline \hline$\gamma_{0}$ & 2.47 \\
$\gamma_{1}$ & 0.39 \\
$\gamma_{2}$ & 0 \\
$\gamma_{3}$ & 0.315 \\
$\gamma_{4}$ & 0 \\
$\gamma_{5}$ & 0 \\
$\Delta$ & 0 \\
\hline \hline
\end{tabular}


In the interval $E_{c}<<|\varepsilon|<<\gamma_{1}$ equations (15), (16), and (17) lead to the approximate formula for the two low-energy bands of electrons and holes in the bilayer

$$
\varepsilon_{e, h}\left(k_{x}, k_{y}\right) \approx \pm \frac{\gamma_{0}^{2}}{\gamma_{1}} \zeta^{2},
$$

that exhibits a quadratic dependence on $k_{\perp}$ discussed in Ref. 22. Here $E_{c}=\left(\gamma_{1} / 4\right)\left(\gamma_{3} / \gamma_{0}\right)^{2}$, and the signs plus and minus correspond to the electrons and holes, respectively.

At energies $|\varepsilon|<E_{c}$ the role of the so-called trigonal warping, which is characterized by the parameter $\gamma_{3}$ in Hamiltonian (16), (17), increases, and this warping breaks the line $\varepsilon=$ constant in the $k_{x}-k_{y}$ plane into one central and three side pockets [22]. We emphasize here that each of these pockets contains a point at which the electron and the hole bands contact, and near all these points the spectrum is linear in k, Fig. 2, see also [24-26]. Thus, in contrast with graphene, in the graphite bilayer near the point $K$ there are four Dirac points. The central contact point coincides with $\mathrm{K}$, while the three side contact points are at a distance of $(\sqrt{3} / 2 \pi)\left(\gamma_{3} \gamma_{1} / \gamma_{0}^{2}\right)|\Gamma K| \approx 0.005|\Gamma K|$ from $\mathrm{K}$ where $|\Gamma K|$ is the distance between the point $K$ and the center $\Gamma$ of the Brillouin zone.

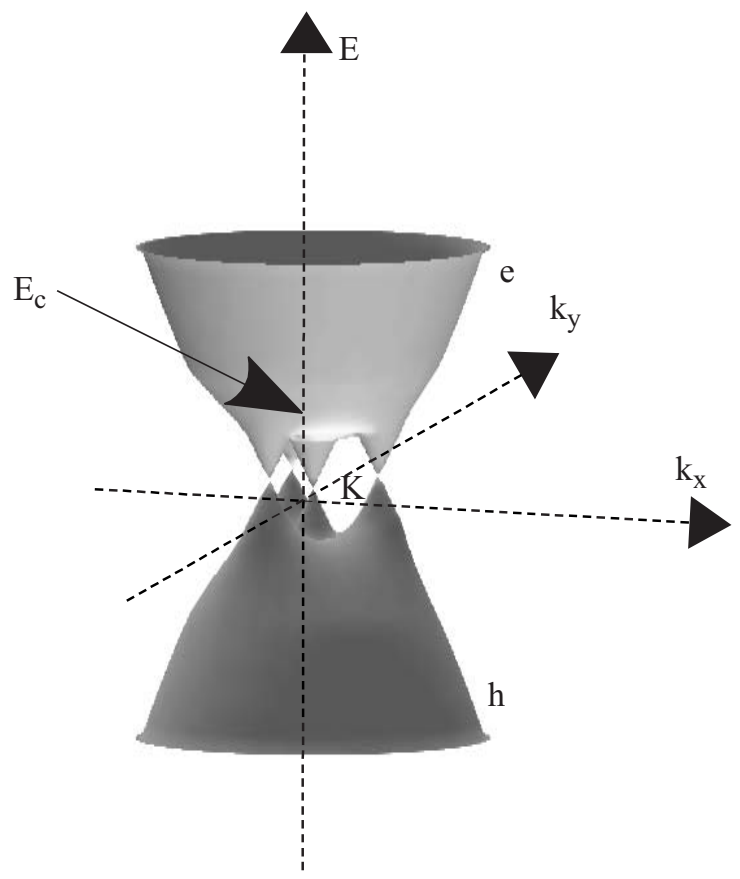

Fig. 2. Shown is the dispersion law $\varepsilon\left(k_{x}, k_{y}\right)$ of the electrons (e) and holes (h) in the graphite bilayer near the point $K$. At the energy $E_{C}$ all the electron cones merge. Note that without neglecting the parameter $\gamma_{4} \approx 0.044 \mathrm{eV}$, the spectrum $\varepsilon\left(k_{x}, k_{y}\right)$ is slightly asymmetric relative to the plane $\varepsilon=0$ : The Dirac points in the central and side cones are at energies $\varepsilon=0$ and $\varepsilon=2 \gamma_{4} \gamma_{1} \gamma_{3}^{2} / \gamma_{0}^{3} \approx$ $\approx 0.15 E_{c}$, respectively. Thus, in contrast with graphene, the graphite bilayer is a semimetal, and changing the Fermi level in it, one cannot obtain the concentration of the charge carriers less than $\sim 10^{8} \mathrm{~cm}^{-2}$. Scales of the plot are distorted for clarity.
The spectrum shown in Fig. 2 clarifies existence of the resistivity maximum discovered in the bilayer [27]. In graphene the universal resistivity maximum $h / 4 e^{2}$ was observed at zero magnetic field and low charge-carrier concentration $N$, and this maximum was explained by absence of localization for electrons with the Dirac-type spectrum [2]. A similar resistivity maximum was also observed in the bilayer [27], and Novoselov et al. [27] emphasized that this observation is unexpected due to the parabolic spectrum in the bilayer. In this context, existence of the Dirac points seems to shed a light on appearance of the resistivity maximum in the bilayer.

McCann and Fal'ko derived the Berry phase for the electron orbits in the bilayer from an effective Hamiltonian that leads to the parabolic spectrum (18). But in the case of the bilayer the trigonal warping generates the three additional side Dirac points and changes the type of the central band-contact point. Thus, for the derivation of the Berry-phase value to be justified, it is necessary to consider the real symmetry of the spectrum in the bilayer and to take into account all the band-contact points of this spectrum.

Since the interaction between carbon atoms in a graphite layer is larger than the interaction between the atoms in different graphite layers (i.e. $\gamma_{0}>\gamma_{i}$ ), we use Hamiltonian (8) of a single layer to include the spin-orbit interaction in Hamiltonian (16), (17) of the bilayer. Then, in the leading order in the small parameter $\Delta_{s o} / \gamma_{1}$ the matrix elements $E_{3}$ in the third and forth lines of formula (16) should be replaced by $E_{3}-\Delta_{s o}$ and $E_{3}+\Delta_{s o}$, respectively.

The calculation of the field $\Omega\left(k_{x}, k_{y}\right)$ in graphite bilayer is similar to the calculation of this quantity in

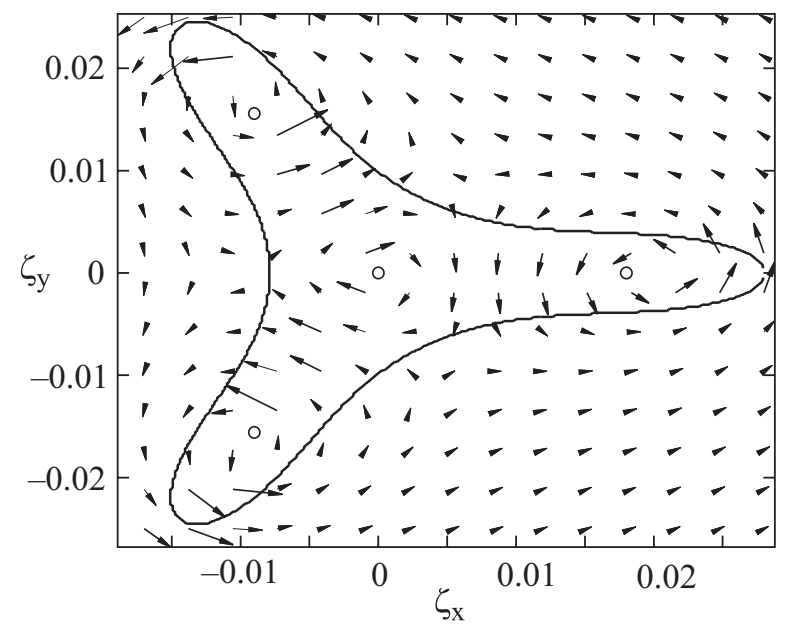

Fig. 3. The field $\Omega\left(k_{x}, k_{y}\right)$ (arrows) for the electron band e in the bilayer. The length of the arrows is proportional to $|\Omega|$. Small circles mark the positions of the Dirac points in the $k_{x}-k_{y}$ plane, and the solid line shows an electron orbit with $\varepsilon>E_{c}$. Note that near the central and the side Dirac points the vector $\Omega$ «circulates» in opposite directions. The components $k_{i}$ are given in the dimensionless units $\zeta_{i}=(2 \pi / \sqrt{3})\left(k_{i} /|\Gamma K|\right)$. 
graphene. Eventually we arrive at the result shown in Fig. 3. Note that near the central and the side Dirac points the vector $\Omega$ «circulates» in opposite directions, which means the opposite signs of the Berry phases generated by these points. The calculation of the integral (1) gives the Berry phase $\pi$ for electron orbits surrounding each of the three side Dirac points and $-\pi$ for an orbit around the central point [12]. Since the Berry phase of an electron does not depend on a size or a shape of its orbit in the Brillouin zone but is determined only by the Dirac points enclosed by the orbit, one finds the Berry phase $3 \pi-\pi=2 \pi$ for electrons with energies $E>E_{c}=\left(\gamma_{1} / 4\right)\left(\gamma_{3} / \gamma_{0}\right)^{2} \approx 2 \mathrm{meV}$ (i.e., at the electron concentration $N>N_{c} \approx 5 \cdot 10^{10} \mathrm{~cm}^{-2}$ ) when their orbits surround all these four points. Thus, at $\varepsilon>E_{c}$ we arrive at the same Berry phase $2 \pi$ as in the case of the parabolic spectrum (18) in spite of the change in the degeneracy of the energy bands. The difference in the Berry phases for the parabolic and real spectra can manifest itself only in the interval $-E_{c}<\varepsilon<E_{c}$.

The coincidence of the Berry phases for the parabolic and the real spectra can be understood as follows: McCann and Fal'ko [22] found Landau levels for spectrum (18) exactly, and they obtained $\varepsilon_{n}=\hbar \omega_{c} \sqrt{n(n-1)}$ where $\omega_{c}=2 v^{2} e H / c \gamma_{1}$ is the cyclotron frequency ( $v$ is proportional to $\gamma_{0}$, see above). The expansion of this expression at large $n \gg 1$ gives $\varepsilon_{n} \approx \hbar \omega_{c}(n-1 / 2)$. Comparing this result with the semiclassical formula $\varepsilon_{n} \approx \hbar \omega_{c}(n+\gamma)$ and taking into account Eq. (4), we obtain $\Phi_{B}=2 \pi$. It is clear that these considerations remain true even with the trigonal warping of the spectrum if this warping is sufficiently small, $E_{c}<<\hbar \omega_{c}$. Thus, we conclude that the Berry phase for the real spectrum has to be equal to $2 \pi$ at least at high energies $\varepsilon \gg \hbar \omega_{c} \gg E_{c}$. But this phase does not depend on $\varepsilon$ in the region $\varepsilon>E_{c}$, and hence the conclusion remains valid everywhere in this region.

The Berry phase $2 \pi$ leads to the half-integer $\gamma$, Eq. (4), as in the case of the usual parabolic spectrum. Hence, one may expect to observe the conventional integer quantum Hall effect in the graphite bilayer. Such the effect was indeed observed [27], but with a specific feature due to the approximate degeneracy of the Landau levels with $n=0$ and $n=1$.

\section{The Berry phase in graphite}

With increasing number of graphite layers, the electron energy structure of such a multilayer evolves to the three-dimensional spectrum of bulk graphite, and the Dirac points (that occur in the bilayer and in multilayers at certain values of $k_{z}$ ) merge and transform into the band-contact lines. According to [25], the multilayers with 10 or a larger number of layers is practically indistinguishable from the bulk material, and now we shall analyze the spectrum of graphite and the Berry phase in it.
Table 2. Values of the parameters of SWM model (eV) that lead to the spectrum of Ref. 22.

\begin{tabular}{c|c}
\hline \hline Parameter & Value \\
\hline$\gamma_{0}$ & $3.16 \pm 0.05$ \\
$\gamma_{1}$ & $0.39 \pm 0.01$ \\
$\gamma_{2}$ & $-0.020 \pm 0.002$ \\
$\gamma_{3}$ & $0.315 \pm 0.015$ \\
$\gamma_{4}$ & $0.044 \pm 0.024$ \\
$\gamma_{5}$ & $0.038 \pm 0.005$ \\
$\Delta$ & $-0.008 \pm 0.002$ \\
$\varepsilon_{F}$ & $-0.024 \pm 0.002$ \\
\hline \hline
\end{tabular}

As it was mentioned above, the electron energy spectrum of graphite is described by the SWM model [23], and values of the parameters of this model were found sufficiently accurately from the analysis of various experimental data; see the review of Brandt et al. [28] and Table 2. In Fig. 4 the electron energy bands of graphite are shown. In the edge HKH of the Brillouin zone of graphite the bands $\varepsilon_{2}(\mathbf{k})$ and $\varepsilon_{3}(\mathbf{k})$ are degenerate. In other words, this edge is the band-contact line. Apart from the

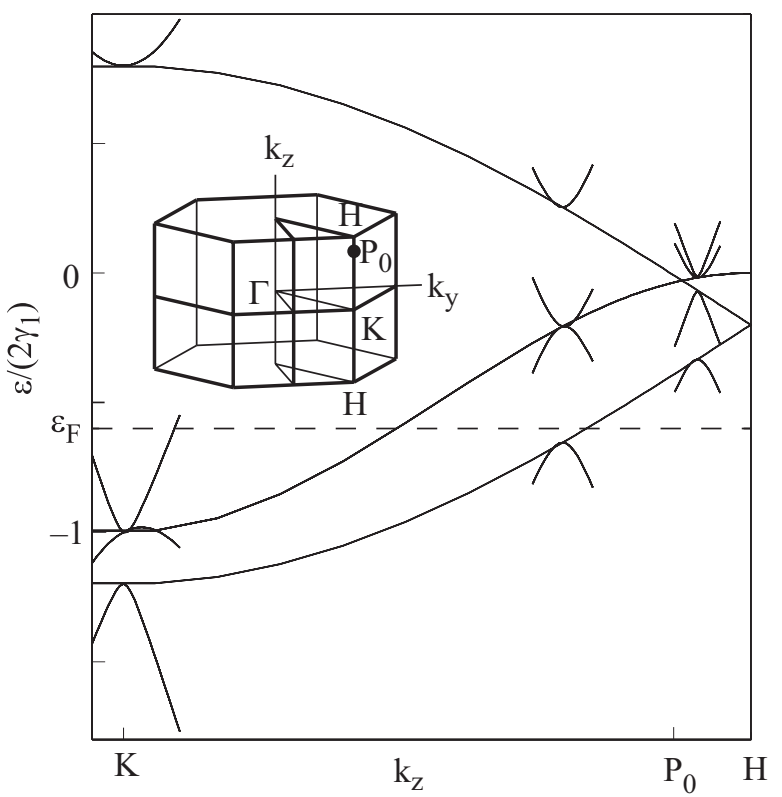

Fig. 4. Dependences of the bands $\varepsilon_{i}(i=1-4)$ on $\mathbf{k}$ near the edge $H K H$ in graphite. Shown are the dependences $\varepsilon_{i}\left(k_{z}\right)$ at $k_{\perp}=0$ and the dependences of $\varepsilon_{i}$ on $k_{\perp}=\sqrt{k_{x}^{2}+k_{y}^{2}}$ at some characteristic values of $k_{z}$. The dashed line marks the position of the Fermi level $\varepsilon_{F}$. For clarity, in the construction of the figure the parameter $\gamma_{1}$ has been used which is twenty times smaller than that of Table 2. For realistic value of $\gamma_{1}$ the point $P_{0}$ is much closer to the point $H$ than in the figure. The insert shows the Brillouin zone of graphite and its characteristic points. 
band-contact line coinciding with the edge $H K H$, three additional band-contact lines of the same bands exist near this edge [29], Fig. 5. The contact in these three side lines is accidental [9]. It is due to the trigonal warping of the spectrum. In the vicinity of the point $K$ the three additional band-contact lines can be approximately found from the equations [30]:

$$
\cos 3 \alpha=1, \quad \zeta \approx \frac{4 \gamma_{3} \gamma_{1}}{\gamma_{0}^{2}} \cos ^{2} \xi
$$

Note that at $\cos \xi=0.5$ these equations give positions of the Dirac points in the bilayer. Thus, one may say that the four band-contact lines in graphite develop from the four Dirac points of the bilayer, Fig. 2.

The bands $\varepsilon_{2}(\mathbf{k})$ and $\varepsilon_{3}(\mathbf{k})$ are degenerate along $K H$ in the interval from $K$ to the point $P_{0}$ defined by the condition $E_{1}(\xi)=E_{3}(\xi)$ (i.e., by the equality $\cos \xi \approx|\Delta| / 2 \gamma_{1} \approx 0.01$ ), while from $P_{0}$ to the point $H$ the degenerate bands are $\varepsilon_{3}(\mathbf{k})$ and $\varepsilon_{4}(\mathbf{k})$. The change of the band degeneracy at $P_{0}$ can be understood if one takes into account an infinitesimal spinorbit interaction in SWM model. Thus, the situation in graphite can be described as follows: The four contact lines of the bands $\varepsilon_{2}(\mathbf{k})$ and $\varepsilon_{3}(\mathbf{k})$ come to the point $P_{0}$ from the one side of the $H K H$ axis, and the four contact lines of the bands $\varepsilon_{3}(\mathbf{k})$ and $\varepsilon_{4}(\mathbf{k})$ come to this point from the opposite side, and all these lines merge at the point $P_{0}$. It is essential that in

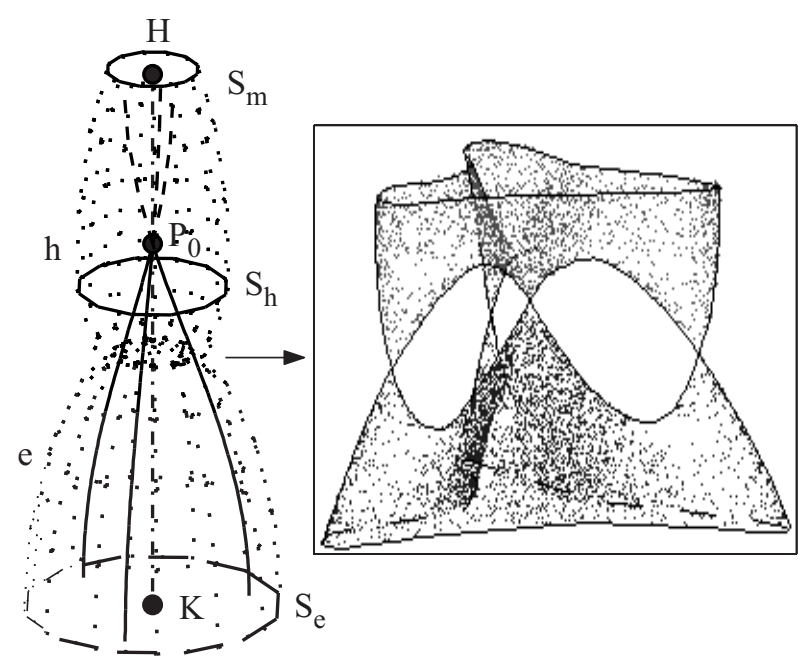

Fig.5. Sketch of the Fermi surface (a half of it) and of the band contact lines in graphite. The accidental contact of the bands $\varepsilon_{2}(\mathbf{k})$ and $\varepsilon_{3}(\mathbf{k})$ occurs along the solid lines, while the dashed lines mark the accidental contact of the bands $\varepsilon_{3}(\mathbf{k})$ and $\varepsilon_{4}(\mathbf{k})$. The same bands are in contact along the HKH axis due to the symmetry of the crystal. All the lines merge at the point $P_{0}$. Shown are also the maximum cross sections of the electron $\left(S_{e}\right)$ and hole $\left(S_{h}\right)$ in the magnetic field along the HKH axis. A part of the Fermi surface where the electron and hole majorities touch is presented in an enlarged scale on the right; the band-contact lines pass through the conical features of the Fermi surface. the vicinity of all these lines the band splitting is linear in a deviation of $\mathbf{k}$ from the lines.

The Fermi surface of graphite consists of elongated pockets enclosing the edge $H K H$ of its Brillouin zone. These pockets are formed by electrons and holes which are located near the points $K$ and $H$ of the Brillouin zone, respectively, see Fig. 5. The electron pocket is formed by electrons of the third band $\varepsilon_{3}(\mathbf{k})$, while the hole group belongs to the second band $\varepsilon_{2}(\mathbf{k})$. All the contact lines of the bands $\varepsilon_{2}(\mathbf{k})$ and $\varepsilon_{3}(\mathbf{k})$ lie under the Fermi surface of the electron and hole groups. When the lines pass from the electron part of the surface to its hole part, the conical features of the Fermi surface, the so-called «outrigger» pieces [28], appear, Fig. 5. The lines are just axes of these four pieces connecting the electron and hole parts. In the magnetic field $H$ parallel to a band-contact line the splitting of the Landau levels for the electron states near the line is proportional to $\sqrt{H}$, see Eq. (14). In the case of graphite these levels are known as the so-called «leg levels» investigated by Dresselhaus [31]. The existence of the leg levels (and, in fact, of the band-contact lines in graphite) was confirmed by magneto-optical experiments $[32,33]$. Interestingly, the well-known large diamagnetism of graphite [34] is also caused by electron states near the band-contact lines [35].

When the magnetic field $H$ is directed along the $z$ axis, the maximum electron cross section in $k_{z}$ is located at $\xi=0$, while the maximum cross section of the holes is between the points $K$ and $P_{0}$, viz., at $\cos \xi \approx \pm\left(\varepsilon_{F} / 6 \gamma_{2}\right)^{1 / 2} \approx 0.45$ where $\varepsilon_{F}$ is the Fermi energy in graphite, see Fig. 5. Thus, both these cross sections are penetrated by the four band-contact lines. The Berry phases of these lines coincide with the Berry phases of the corresponding Dirac points in the graphite bilayer. Hence, for both the extremal cross sections of graphite the Berry phase is $2 \pi$, and one can expect to find the usual half-integer $\gamma$ in the oscillation effects. Such the value of $\gamma$ was indeed observed in Refs. 36,37.

1. A.K. Geim, K.S. Novoselov, Nature Materials 6, 183 (2007).

2. K.S. Novoselov, A.K. Geim, S.V. Morozov, D. Jiang, M.I. Katsnelson, I.V. Grigorieva, S.V. Dubonos, and A.A. Firsov, Nature 438, 197 (2005).

3. Y. Zhang, Y-W. Tan, H.L. Stomer, and P. Kim, Nature 438, 201 (2005).

4. M.V. Berry, Proc. Roy. Soc. London A392, 45 (1984).

5. G.P. Mikitik and Yu.V. Sharlai, Phys. Rev. Lett. 82, 2147 (1999).

6. G.P. Mikitik and Yu.V. Sharlai, Fiz. Nizk. Temp. 22, 762 (1996) [Low Temp. Phys. 22, 585 (1996)].

7. S.G. Sharapov, V.P. Gusynin, and H. Beck, Phys. Rev. B69, 075104 (2004).

8. J. Zak, Phys. Rev. Lett. 62, 2747 (1989).

9. C. Herring, Phys. Rev. 52, 365 (1937).

10. Y. Aharonov and D. Bohm, Phys. Rev. 115, 485 (1959).

11. G.P. Mikitik and Yu.V. Sharlai, Zh. Eksp. Teor. Fiz. 114, 1375 (1998) [JETP 87, 747 (1998)]. 
12. G.P. Mikitik and Yu.V. Sharlai, Phys. Rev. B77, 113407 (2008).

13. E.I. Blount, Solid State Physics, vol. 13, Academic Press, New York and London (1962), p. 305

14. L. Onsager, Philos. Mag. 43, 1006 (1952).

15. I.M. Lifshitz and A.M. Kosevich, Zh. Eksp. Teor. Fiz. 29, 730 (1955) [Sov. Phys. JETP 2, 636 (1956)].

16. G.P. Mikitik and Yu.V. Sharlai, Phys. Rev. B65, 184426 (2002).

17. D. Shoenberg, Magnetic Oscillations in Metals, Cambridge University Press, Cambridge, England (1984).

18. I.A. Luk'yanchuk and Y. Kopelevich, Phys. Rev. Lett. 93, 166402 (2004).

19. C.K. Kane and E.J. Mele, Phys. Rev. Lett. 95, 226801 (2005).

20. N.A. Sinitsyn, J.E. Hill, H. Min, J. Sinova, and A.H. MacDonald, Phys. Rev. Lett. 97, 106804 (2006).

21. In general case when $\Delta_{S O}$ differs from zero the Landau level spacing is determined by the electron $g$ factor. Interestingly, the sum of the intraband and the interband contributions to this $g$ factor is independent of $\Delta_{S o}$ [11]. This means that the Landau level spacing is identical to that for $\Delta_{s o}=0$. That is why we use $\gamma=0$ in Eq. (14). Here we also neglect the Zeeman term $\pm \mu_{B} H$ associated with the direct interaction of the electron spin with the magnetic field since this term is much smaller than the Landau level spacing; $\mu_{B}$ is the Bohr magneton.

22. E. McCann and V. Fal'ko, Phys. Rev. Lett. 96086805 (2006).

23. J.C. Slonzewski and P.R. Weiss, Phys. Rev. 109, 272 (1958); J.W. McClure, Phys. Rev. 108, 612 (1957).
24. M. Koshino and T. Ando, Phys. Rev. B73, 245403 (2006).

25. B. Partoens and F. M. Peeters, Phys. Rev. B74, 075404 (2006).

26. J.L. Manes, F. Guinea, and M.A.H. Vozmediano, Phys. Rev. B75, 155424 (2007).

27. K.S. Novoselov, E. McCann, S.V. Morozov, V.I. Fal'ko, M.I. Katsnelson, U. Zeitler, D. Jiang, F. Schedin, and A.K. Geim, Nature Phys. 2, 177 (2006).

28. N.B. Brandt, S.M. Chudinov, and Ya.G. Ponomarev, Semimetals I. Graphite and its Compouds, Elsevier, Amsterdam, (1988).

29. G.P. Mikitik and Yu.V. Sharlai, Phys. Rev. B73, 235112 (2006).

30. These equations in Ref. [29] contain misprints.

31, G. Dresselhaus, Phys. Rev. B10, 3602 (1974).

32. D.A. Platts, D.D.L. Chung, M.S. Dresselhaus, Phys. Rev. B15, 1087 (1977).

33. R.E. Doezema, W.R. Datars, H. Schaber, and A. Van Schyndel, Phys. Rev. B19, 4224 (1979).

34. J.W. McClure, Phys. Rev. 101, 1642 (1956); ibid 119, 606 (1960); M.P. Sharma, L.C. Johnson, and J.W. McClure, Phys. Rev. B9, 2467 (1974).

35. G.P. Mikitik and I.V. Svechkarev, Fiz. Nizk. Temp. 15, 295 (1989) [Sov. J. Low Temp. Phys. 15, 165 (1989)].

36. D.E. Soule, J.W. McClure and L.B. Smith, Phys. Rev. 134, A453 (1964).

37. S.J. Williamson, S. Foner, and M.S. Dresselhaus, Phys. Rev. 140, A1429 (1965). 